\title{
Biomimetic Mechanism of Action of Fluoridated Toothpaste Containing Proprietary REFIX Technology on the Remineralization and Repair of Demineralized Dental Tissues: An In Vitro Study
}

\author{
Fabiano Vieira Vilhena' Sonia Mariza Luiz de Oliveira² Marcelo Henrick Maia Matochek ${ }^{2}$ \\ Pedro Luiz Santos Tomaz ${ }^{2}$ Thales de Sá Oliveira ${ }^{2}$ Paulo Henrique Perlatti D’Alpino ${ }^{2,3, \odot}$
}

\author{
${ }^{1}$ Trials Pesquisa e Desenvolvimento, Bauru, SP, Brasil \\ ${ }^{2}$ Graduate Program in Biotechnology and Health Innovation, \\ Universidade Anhanguera de São Paulo (UNIAN-SP), São Paulo, Brazil \\ ${ }^{3}$ Graduate Program in Science Teaching and Health, Universidade \\ Anhanguera de São Paulo (UNIAN-SP), São Paulo, Brazil
}

Address for correspondence Paulo Henrique Perlatti D’Alpino, DDS, MSc, PhD, Anhanguera University of São Paulo-UNIAN SP, Graduate Program in Biotechnology and Health Innovation, Avenue Raimundo Pereira de Magalhães, 3.305, São Paulo, SP, CEP: 05145200, Brazil (e-mail: paulodalpino@gmail.com).

Eur J Dent 2021;15:236-241

\begin{abstract}
Keywords

- enamel

- dentin

- tooth remineralization

- toothpaste

- microscopy

- electron

- scanning

Objectives This in vitro study aimed to characterize the mineral content and surface and cross-sectional morphology of enamel and dentin tissues treated with a $1450 \mathrm{ppm}$ fluoride-containing toothpaste with REFIX technology.

Materials and Methods Bovine enamel blocks $(n=5)$ were obtained $(4 \times 4 \times 6 \mathrm{~mm})$, demineralized (artificial caries lesion), and treated ( $\mathrm{pH}$ cycling and brushing with the toothpaste). During the $\mathrm{pH}$ cycling, which lasted for 7 days (demineralization and remineralization took 6 and 18 hours, respectively), the enamel was brushed for 5 minutes using an electric toothbrush before being immersed in a remineralizing solution. The dentin blocks were acid-etched for 2 minutes ( $0.05 \mathrm{M}$ citric acid, $\mathrm{pH} 1.8$ ) to expose the dentinal tubules $(n=5)$. Morphological analysis of the dentin was performed immediately and after 7 days of brushing with the dentifrice, and compared with the control group. The specimens were then cross-sectioned. The surface and cross-sectional micromorphology were assessed using scanning electron microscopy (SEM). The elemental analyses (weight\%) were determined with an energy-dispersive $\mathrm{X}$-ray spectroscopy (EDS).

Results The toothpaste with REFIX technology remineralized and repaired the surface enamel effectively. The elemental analysis also demonstrated that treating the enamel with the toothpaste formed a silicon-enriched mineral layer on the enamel surface. Elemental analysis of the enamel cross-sections showed that the toothpaste induced a mineral change. The results were also consistent in the dentin, where the dentinal tubules were progressively occluded until there was complete occlusion after 7 days.

Conclusions We prove the biomimetic mechanism of action of fluoridated toothpaste containing proprietary REFIX technology for obtaining silicon-enriched, remineralized and repaired dental tissues.
\end{abstract}

published online November 26, 2020
DOI https://doi.org/ $10.1055 / \mathrm{s}-0040-1716781$ ISSN 1305-7456. (c) 2020. European Journal of Dentistry.

This is an open access article published by Thieme under the terms of the Creative Commons Attribution-NonDerivative-NonCommercial-License, permitting copying and reproduction so long as the original work is given appropriate credit. Contents may not be used for commercial purposes, or adapted, remixed, transformed or built upon. (https://creativecommons.org/licenses/by-nc-nd/4.0/) Thieme Medical and Scientific Publishers Pvt. Ltd., A-12, 2nd Floor, Sector 2, Noida-201301 UP, India 


\section{Introduction}

Although there are toothpastes with specific indications on the market, multipurpose toothpastes have been launched with different therapeutic effects. ${ }^{1}$ In spite of the varied reasons for choosing a toothpaste (brand name, taste, price, package, easy to buy, dentist's indication, among others), consumers are prone to choosing an all-in-one product to reduce dental hypersensitivity and promote enamel remineralization. ${ }^{1}$ The effectiveness of these toothpastes in promoting dentin tubule occlusion and remineralization depends not only on the fluoride content but also on the presence of other active ingredients. ${ }^{2}$ The addition of different components or supplements in combination with fluoride, known as the biomimetic approach, represents an alternative mechanism that reproduces the natural process of dental tissue mineralization. ${ }^{3.4}$ In this manner, several commercially available oral care products, containing different active ingredients associated with fluoride, claim to boost the remineralization and regeneration potential of these formulations. ${ }^{5,6}$

The remineralization process can be enhanced by the substitution of small levels of ionic species in the different sites of the hydroxyapatite molecule. ${ }^{7}$ Each ionic grouping of the hydroxyapatite molecule can be replaced by another ionic group of the same or different valence. ${ }^{8}$ Changes in the hydroxyapatite solubility may occur, depending on the substitution at the different sites (calcium, phosphate, and hydroxyl sites). ${ }^{8}$ Fluorine ions (F-) can replace hydroxyl ions $(\mathrm{OH}-)$, and this substitution tends to reduce hydroxyapatite solubility. ${ }^{9}$ Substituting silicon, or silicate, into the remineralizing hydroxyapatite enhances the bioactivity and apatite-forming ability of hydroxyapatite. ${ }^{10,11}$ Although it is claimed that these changes occur mainly at the hydroxyapatite surface, ${ }^{12}$ it seems to be the reason for retaining fluoride in the composition, even at lower concentrations, against the risk of fluorosis. ${ }^{13}$ In this manner, balanced compositions in these all-in-one formulations are of imperative importance.

Recently, a proprietary technology named REFIX was developed. It comprises a fluoride-containing toothpaste in association with phosphates and silica. According to the manufacturer, this association favors the formation of a fluoridated apatite and the deposition of silicate, which is also incorporated deep into the hydroxyapatite and onto the open dentinal tubules. A previous in vitro study ${ }^{14}$ reported that the REFIX-containing toothpaste could remineralize within the subsurface carious lesion effectively when compared with other commercial $1450 \mathrm{ppm}$ fluoride-containing toothpastes. A clinical trial evaluated the efficacy of a toothpaste containing REFIX technology against dentin hypersensitivity. ${ }^{15}$ The authors proved that this product was effective against dentin hypersensitivity, achieving the result after 1 week of consistent use. ${ }^{15}$

To date, the mechanism with which the REFIX technology mineralizes enamel and reduces dentin hypersensitivity is unclear. The purpose of this in vitro study was to characterize the mineral content and surface morphology of dental tissues treated with fluoridated toothpaste containing REFIX technology to understand the mechanism of action of this oral care product.

\section{Materials and Methods}

\section{Specimen Preparation of Dental Enamel}

Enamel blocks $(4 \times 4 \times 6 \mathrm{~mm})$ were obtained from bovine incisors using a diamond-impregnated disc (Extec, Enfield, CT, USA) under water lubrication in a cutting machine (IsoMet 1000; Buehler, Lake Bluff, IL, USA). The enamel blocks $(n=5)$ were then embedded in epoxy resin (EpoxiCure Epoxy Resin and Hardener, Buehler, Lake Bluff, IL, USA). Surfaces were then wet-polished with 600 -grit SiC paper at low speed and with 1,200-grit SiC paper at high speed using a polishing machine (Single Grinder Polisher, Buehler, Lake Bluff, IL, USA). The final polishing was performed with $1 \mu \mathrm{m}$ diamond paste and wet felt wheels (Buehler, Lake Bluff, IL, USA).

The enamel blocks were immersed in $30 \mathrm{~mL}$ of $50 \mathrm{mM}$ acetate buffer solution containing $3 \mathrm{mM} \mathrm{CaCl} 2 \cdot \mathrm{H}_{2} \mathrm{O}, 3 \mathrm{mM}$ $\mathrm{KH}_{2} \mathrm{PO}_{4}, 50 \mathrm{mM}$ lactic buffer, and $6 \mu \mathrm{M}$ methylhydroxydiphosphate (MHDP), at $\mathrm{pH} 5.0$ for 5 days to demineralize the enamel and produce caries-like lesions. ${ }^{16}$ After demineralization, the enamel blocks underwent $\mathrm{pH}$ cycling by interchanging a demineralization solution $\left(1.5 \mathrm{mM} \mathrm{CaCl}_{2}, 0.9 \mathrm{mM}\right.$ $\mathrm{KH}_{2} \mathrm{PO}_{4}, 50 \mathrm{mM}$ acid lactic buffer, $\mathrm{pH}$ 5.0, 8 hour) and remineralization solution $\left(5 \mathrm{mM} \mathrm{CaCl}_{2}, 0.9 \mathrm{mM} \mathrm{KH}_{2} \mathrm{PO}_{4}, 130 \mathrm{mM}\right.$ $\mathrm{KCl}, 20 \mathrm{mM}$ HEPES, $5 \mathrm{mM} \mathrm{NaN}_{3}$, $\mathrm{PH} 7.0,16$ hour) for 7 days. ${ }^{17}$ During the $\mathrm{pH}$ cycling, the enamel surfaces were treated with $30 \mathrm{~mL}$ toothpaste slurry (3:1 weight ratio of deionized water to toothpaste) with an electric toothbrush (Oral-B Vitality Precision Clean rechargeable toothbrush; Procter \& Gamble, Cuajimalpa, Mexico). Brushing was performed between the incubation with the remineralization and demineralizing solutions. The enamel blocks were brushed with the toothpaste slurry ( $2 \mathrm{~mL} / \mathrm{block})$ using the electric toothbrush for 5 minutes $\left(120 \text { cycles per minute, } 22^{\circ} \mathrm{C}\right)^{14}$ three times a day for 1 week. The toothbrushes were fixed in a device that allowed the heads to be aligned parallel to the specimens' surfaces. ${ }^{18}$ The specimens were brushed in linear movements with an axial load of $200 \mathrm{~g}$. Between the steps, the specimens were water rinsed with deionized water for 5 seconds.

\section{Specimen Preparation of Dental Dentin}

Bovine dentin blocks were obtained $(n=5)$ as described earlier. The dentin blocks were embedded in epoxy resin, and the surfaces were wet-polished with 600-grit SiC paper at low speed and with 1,200-grit SiC paper at high speed using a polishing machine. The specimens were acid-etched for 2 minutes ( $0.05 \mathrm{M}$ citric acid, $\mathrm{pH} 1.8$ ) to remove the smear layer and expose the dentinal tubules. ${ }^{19}$ The experimental groups for the dentin analysis were as follows: I, control, untreated group; II, morphological analysis immediately after brushing with the dentifrice; III, morphological analysis after 7 days of brushing with the dentifrice..$^{15}$

Characterization of the enamel and dentin surfaces and cross-sections by scanning electron microscopy (SEM) imaging observation and energy-dispersive X-ray spectroscopy (EDS) 
The morphological analysis of the specimens was performed using SEM (TESCAN VEGA3; LMU, Kohoutovice, the Czech Republic) operating at $15 \mathrm{kV}$. The blocks were first sputter-coated with gold in a vacuum evaporator (MED 010; Balzers, Balzers, Liechtenstein), and then microscopically analyzed to obtain photomicrographs of the superficial morphology of the treated specimens ( $\times 1,000$ magnification). Representative images of selected regions of the specimens were obtained for characterizing the morphological aspect of the surface. The EDS point analysis $\left(80 \mathrm{~mm}^{2}\right.$, SDD [silicon drift detector], Oxford Instruments, Concord, MA, USA) was performed to determine a qualitative elemental analysis of the specimens, operating in high-vacuum mode and an accelerating voltage of $15 \mathrm{kV}$. Five points per sample were randomly selected ( $300 \mu \mathrm{m}^{2}$ per point), and the mean values were calculated.

For the subsurface analysis, cross-sections of the bovine blocks were obtained by longitudinally sectioning the specimens under water cooling. Both half-blocks were used for the SEM and elemental analyses. The halves were dehydrated in silica gel for 3 hours. The specimens were then gold-sputtered and evaluated using SEM coupled with EDS.

\section{Results}

-Fig. 1 shows the representative scanning electron micrographs of the enamel. - Fig. 1A demonstrates the clearly different areas, separated by the dotted line: the area above the line represents the control demineralized area; the area below the line represents the remineralized treated area.
-Fig. 1B and 1C show the representative scanning electron micrographs at higher magnification of both areas. The ability of the REFIX-containing toothpaste to remineralize and regenerate the highlighted area was clearly demonstrated (-Fig. 1C) in comparison to that observed in the demineralized area (-Fig. 1B). The treated regenerated enamel had smoother surface morphology in comparison to the demineralized area, which presented depressions induced by the caries-like lesions. - Fig. 1D shows a cross-sectional area of the control demineralized enamel; for comparison, - Fig. 1E shows a representative scanning electron micrograph of the remineralized cross-sectional area. In the same way, the morphological differences between these two areas are clearly demonstrated, in which a mineralized layer formed on the enamel surface after $\mathrm{pH}$ cycling interspersed with the

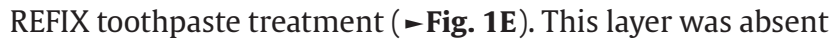
in the control demineralized area ( - Fig. 1D).

-Fig. 2 shows the representative scanning electron micrographs of the enamel cross-sections and the respective elemental mapping at different depths (from the superficial area A to the deepest area D). EDS detected stronger silicon signals at the surface areas A and B (0.56 weight\%), which increased right below the mineralized layer formed at the enamel surface (C, 0.77 weight\%), and then decreased in the deepest area (D, 0.24 weight\%). The amounts of sodium, phosphorus, and calcium also varied as a function of the enamel depths.

-Fig. 3 shows scanning electron micrographs of the morphological analysis of the superficial and cross-sectional areas of the dentin. - Fig. $\mathbf{3 A}$ shows a scanning electron micrograph of the demineralized area with opened dentin tubules.
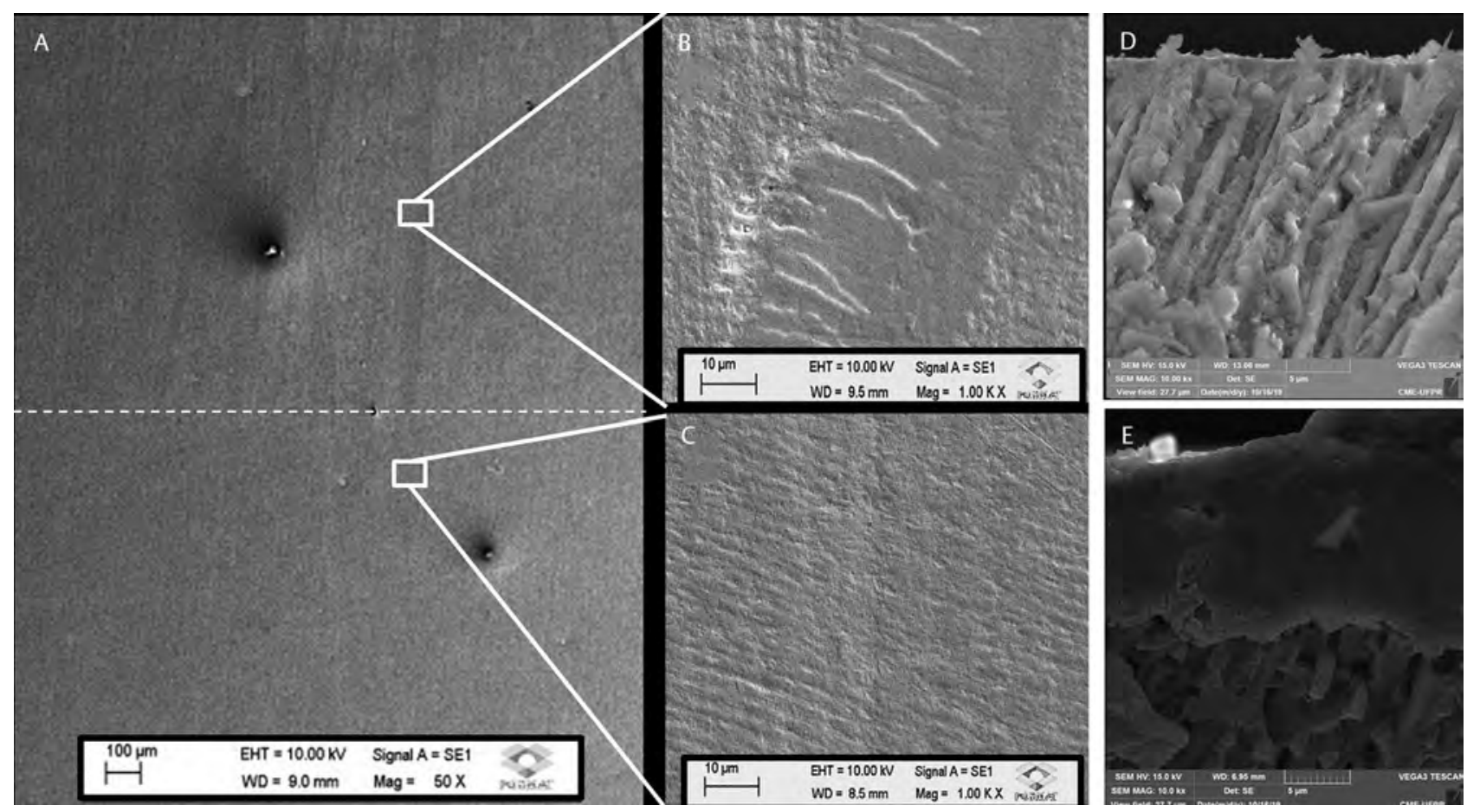

Fig. 1 (A) shows a representative scanning electron micrograph of the enamel surface (magnification 50x); above the line represents the control demineralized area; the area below the line represents the remineralized treated area. (B) shows a representative area of the demineralized enamel. (C) shows a representative area in which the enamel was remineralized and regenerated. (D) shows the cross-sectional area of the control, demineralized enamel half. (E) shows a representative scanning electron microscope (SEM) photomicrograph image the cross-section of enamel block in which a mineralized layer is formed at the treated enamel surface. 
-Fig. 3B shows that a remineralized area is formed immediately after brushing with the toothpaste, exhibiting partially occluded dentin tubules. Conversely, - Fig. 3C shows an area of the dentin surface in perspective, in which a mineralized layer was formed after 7 days of brushing; a protective layer

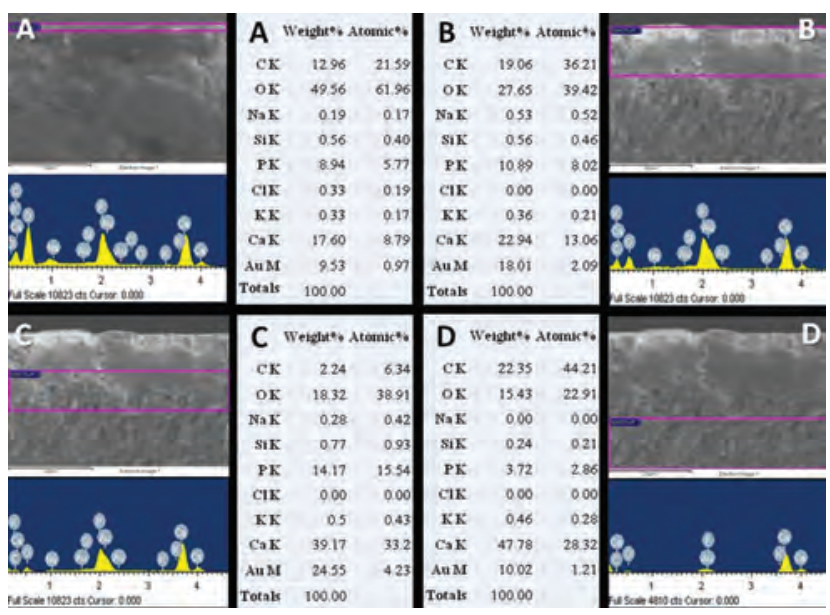

Fig. 2 Representative scanning electron micrographs of the enamel cross-sections and the elemental mapping at different depths (from surface area $A$ to the deepest area D). EDS detected stronger silicon signals at the surface area (0.56 weight\%), which increased right below the mineralized layer formed at the enamel surface (C, 0.77 weight\%), and decreased in the deepest area (D, 0.24 weight\%). The amounts of sodium, phosphorus, and calcium also varied as a function of the depth. on the dentin surface of up to $3 \mu \mathrm{m}$ was noted after 7-day brushing. - Fig. 3D shows the same treated dentin area after 1 week of treatment, with a majority of occluded tubules. -Fig. 3E shows a scanning electron micrograph of the mineralized layer formed at the dentin surface associated with the elemental analysis, which demonstrated the presence of Si (6.70 weight\%), F (0.90 weight\%), Ca (1.60 weight\%), and $\mathrm{P}$ (0.46 weight\%).

\section{Discussion}

The toothpaste tested in the present study contains proprietary REFIX technology, which according to the manufacturer, represents a novel multifunctional phosphate-based dental gel technology in an acidified stabilized phosphate/fluoride complex, which is established especially in saliva. ${ }^{14}$ This combination with saliva and dental tooth structures favors the generation of new minerals containing calcium/phosphate/ fluorine, promoting the enamel surface and remineralizing within the subsurface carious lesion. ${ }^{14}$ This product presents an acid $\mathrm{pH}$ as a function of the proposed technology, whose action is possibly more effective due to the formation of calcium phosphate crystals in an acidic environment, which appears to be the main reason for its effectiveness. ${ }^{14,15}$

The difference observed in the elemental mapping demonstrates that the REFIX toothpaste could modify the structure of the demineralized enamel. It was clearly demonstrated that a remineralized layer formed on the enamel
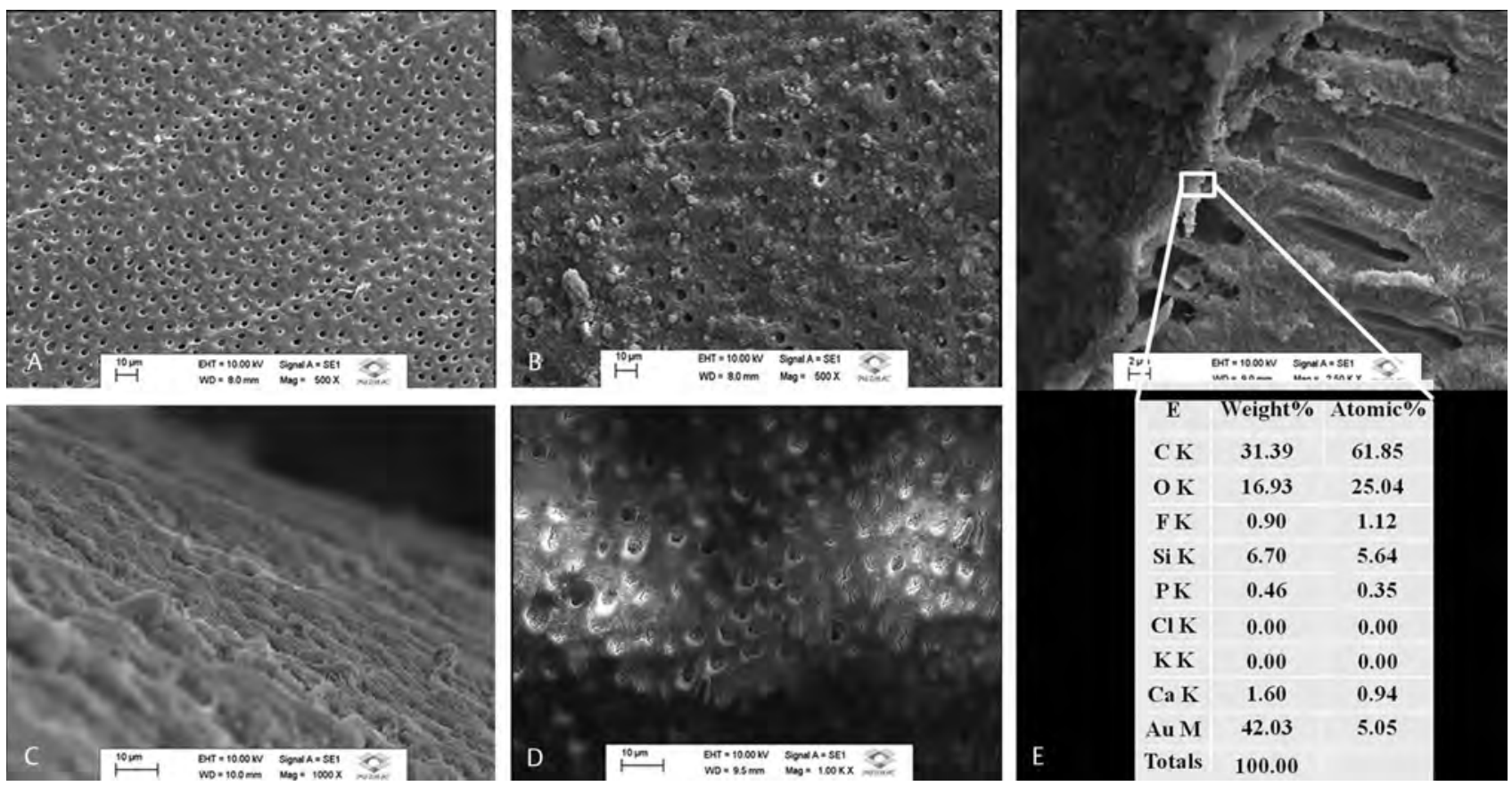

Fig. 3 Scanning electron micrographs of the morphological analysis of the surface and cross-sectional areas of the dentin: (A) shows a representative scanning electron micrograph of the demineralized dentin with open tubules. (B) shows a representative scanning electron micrograph of the dentin immediately after brushing with the toothpaste with occluded dentinal tubules. Figure 3 (c) shows the dentin surface in which a mineralized layer was formed after 7 days of treatment. (D) shows the scanning electron micrograph of the treated dentin with a majority of occluded tubules after 1 week of treatment. (E) shows a scanning electron micrograph of the mineralized layer formed at the dentin surface, demonstrating the presence of silicon (6.70 weight\%), fluorine (0.90 weight\%), calcium (1.60 weight\%), and phosphorus (0.46 weight\%) in the elemental analysis. 
surface after $\mathrm{pH}$ cycling. The elemental mapping demonstrated the presence of silicon deeper in the enamel, with higher concentrations right below the mineralized layer formed on the enamel surface ( $\boldsymbol{- \text { Fig. }} \mathbf{1}$ ). This silicon-enriched hydroxyapatite present in the mineral layer formed on the enamel surface was induced by the REFIX-based toothpaste, which favored the formation of complexes of the bioactive particles calcium, phosphorus, and sodium, as demonstrated in - Fig. 2. Bioactive glass is claimed to induce faster capitation of sodium, calcium, and phosphorus, thus boosting the precipitation of minerals for remineralizing the enamel surface or for reducing the effects of demineralization..$^{20}$ In this way, silicon is regarded as a component of bioactive glass, inducing the formation of nucleation sites for the precipitation of calcium and phosphate ions in the formation of hydroxyapatite. ${ }^{21}$ Calcium silicate is then formed, which is responsible for boosting the mineralization processes of saliva via hydroxyapatite nucleation and mineral formation. ${ }^{22}$ Silicon is claimed to promote more rapid release of $\mathrm{Ca}$ and $\mathrm{P}^{23}$ and induce low negative zeta potential in the biological medium, which has important in vivo effects. ${ }^{21}$ As previously reported, substituting phosphate ions $\left(\mathrm{PO}_{4}\right)$ with silicate ions $\left(\mathrm{SiO}_{4}\right)$ in hydroxyapatite structures to form calcium silicate also confers a protective effect by inducing the formation of hydroxyapatite after its deposition onto the eroded surfaces. ${ }^{24}$ This is particularly due to the induced bioactivity and apatite-forming ability of hydroxyapatite, which is enhanced by the substitution of silicon, or silicate, into the remineralizing hydroxyapatite. ${ }^{10,11}$

Another component of the toothpaste, according to the manufacturer, is the tetrasodium pyrophosphate in association with sodium fluoride $(1450 \mathrm{ppm}) .^{14}$ The aim of oversaturating the enamel surface with calcium and phosphate is to contribute to the decrease in ions on the previously demineralized enamel surface, consequently increasing the resistance to the cariogenic process in the presence of fluoride ions. ${ }^{25} \mathrm{An}$ in vitro study ${ }^{14}$ investigated the remineralization potential of $1450 \mathrm{ppm}$ fluoride-containing toothpastes containing different active remineralization agents after cariogenic challenge with pH cycling. Although the REFIX toothpaste could not recover the hardness of the remineralized enamel surface completely in comparison to the intact enamel (94\%), the product significantly reversed the lesion formed at the subsurface to a depth of around $60 \mu \mathrm{m}$. The mean lower hardness of the remineralized superficial enamel as compared with the intact enamel can be explained by the fact that replacing $\mathrm{PO}_{4}$ with $\mathrm{SiO}_{4}$ seems to lead to decreased crystallinity and thermal stability, and also to increased solubility of the hydroxyapatite structure. ${ }^{26}$ Substituting $\mathrm{PO}_{4}$ with $\mathrm{SiO}_{4}$ is believed to affect the mechanical properties of the silicon-enriched hydroxyapatite in a dose-dependent manner, decreasing hardness and the elastic modulus. ${ }^{27}$ Conversely, as previously noted, the silicon content in the toothpaste formulation in association with fluorine and phosphate groups induces increased bioactivity and apatite-forming ability of hydroxyapatite, which is enhanced by the substitution of silicon, or silicate, into the remineralizing hydroxyapatite..$^{10,11}$
The REFIX-containing toothpaste could also remineralize the dentin tissue effectively by depositing a mineral layer, thus progressively occluding the dentinal tubules. Depositing phosphate and calcium ions at the surface of exposed tubules forms a protective layer against dentinal hypersensitivity ${ }^{28}$ which is an effective treatment. ${ }^{29} \mathrm{~A}$ clinical trial that evaluated the effectiveness of the REFIX-based toothpaste in 53 participants found that the participants experienced a significant reduction in dentin hypersensitivity after 1 week of use. ${ }^{15}$ The REFIX-containing toothpaste reduced $88.3 \%$ of the dentin hypersensitivity after 1 week of use. The authors speculated that the reduction could have been due to the formation of enamel-like deposited crystals, which would reduce the functional area of the dentinal tubules by partially occluding the tubule openings, reducing the fluid flow and, consequently, the dentin hypersensitivity. ${ }^{15}$ In that study, the authors also hypothesized that phosphate-mediated calcium fluoride and calcium silicate crystals would be formed and then deposited on the dentin surface and/or inside the dentinal tubules. Its effect against dentin hypersensitivity was possible since the first use, which corroborates the findings of the present study. Here, a remineralized area formed immediately after brushing with the toothpaste, exhibiting partially occluded dentin tubules ( - Fig. 3B). After 1 week of use, the majority of tubules were occluded ( - Fig. 3D). The elemental analysis also demonstrated the presence of silicon ( 6.70 weight \%) in the remineralized area. The elemental analysis also detected fluorine (0.90 weight\%), calcium (1.60 weight\%), and phosphorus (0.46 weight\%) (-Fig. 3E).

An in vitro study ${ }^{24}$ evaluated the remineralizing effects of calcium silicate deposition on the properties of acid-eroded dental surfaces. Calcium silicate could confer a protective effect by inducing the formation of silicon-enriched hydroxyapatite after its deposition onto the eroded surfaces. ${ }^{24}$ Other studies have also demonstrated the promising effects of silica-based compounds for obliterating dentin tubules. . $^{30,31}$ These results are associated with the ability of silicon-containing oral care products to promote infiltration and subsequent remineralization in order to occlude the dentinal tubules. ${ }^{32}$ This is possible due to ability of silica to adhere and occlude tubules via hydroxyl groups, which aid silica binding with the calcium receptors on the dentin surface. ${ }^{31,32}$ This helps to explain the effectiveness of REFIX toothpaste for inducing dentinal tubule occlusion.

The present study has characterized the mineral content and surface morphology of enamel and dentin tissues treated with $1450 \mathrm{ppm}$ fluoride-containing toothpaste along with REFIX technology. Considering its limitations, the present study could be performed with human teeth instead of bovine teeth. In addition, other toothpastes could be used as a gold standard for comparing the results. In spite of this concern, a control untreated group was used for comparison. Despite the limitations of the present in vitro study, the results of previous studies ${ }^{14,15}$ corroborate our results. Further research is needed to investigate the performance of toothpaste containing REFIX technology in in situ and in vivo studies on the effectiveness of the toothpaste on dental substrates. 


\section{Conclusion}

The 1450 ppm fluoride-containing toothpaste with REFIX technology can remineralize and repair the surface enamel effectively. Elemental analysis demonstrated that treating the enamel with the toothpaste forms a silicon-enriched mineral layer on the enamel surface. In addition, elemental analysis of enamel cross-sections showed that the toothpaste could induce a mineral change. The results are consistent with that of the dentin specimens, where the dentinal tubules were progressively occluded until there was complete occlusion after 7 days of application, corroborating the results of previous studies. A silicon-enriched layer was also formed, occluding the dentinal tubule openings. Further studies are needed to evaluate the resistance of the layer formed on the dental tissue surface against acid challenges for maintaining oral health.

\section{Conflict of Interest}

None declared.

\section{References}

1 Cury JA, Tenuta LM. Evidence-based recommendation on toothpaste use. Braz Oral Res 2014;28(Spec No) :1-7

2 Farooq I, Moheet IA, AlShwaimi E. In vitro dentin tubule occlusion and remineralization competence of various toothpastes. Arch Oral Biol 2015;60(9):1246-1253

3 Xiao Z, Que K, Wang H, et al. Rapid biomimetic remineralization of the demineralized enamel surface using nano-particles of amorphous calcium phosphate guided by chimaeric peptides. Dent Mater 2017;33(11):1217-1228

4 Elgamily H, Safwat E, Soliman Z, Salama H, El-Sayed H, Anwar M. Antibacterial and remineralization efficacy of casein phosphopeptide, glycomacropeptide nanocomplex, and probiotics in experimental toothpastes: an in vitro comparative study. Eur J Dent 2019;13(3):391-398

5 Cardoso CdeA, Lacerda B, Mangueira DF, et al. Mechanisms of action of fluoridated acidic liquid dentifrices against dental caries. Arch Oral Biol 2015;60(1):23-28

6 Kraivaphan P, Amornchat C. Comparative clinical efficacy of three toothpastes in the control of supragingival calculus formation. Eur J Dent 2017;11(1):94-98

7 Vallet-Regí M, Arcos D. Silicon substituted hydroxyapatites. A method to upgrade calcium phosphate based implants. J Mater Chem 2005;15:1509-1516

8 Palard M, Champion E, Foucaud S. Synthesis of silicated hydroxyapatite Ca10(PO4)6-x(SiO4)x(OH)2-x. J Sol 2008;181:1950-1960

9 Yao F, LeGeros JP, LeGeros RZ. Simultaneous incorporation of carbonate and fluoride in synthetic apatites: Effect on crystallographic and physico-chemical properties. Acta Biomater 2009;5(6):2169-2177

10 Gibson IR, Huang J, Best SM, Bonfield W, eds. Enhanced in vitro cell activity and surface apatite layer formation on novel silicon-substituted hydroxyapatites. 12th International Symposium on Ceramics in Medicine; 1999; Nam, Japan: World Scientific Publishing Co. Pte. Ltd

11 Carrouel F, Viennot S, Ottolenghi L, Gaillard C, Bourgeois D. Nanoparticles as anti-microbial, anti-inflammatory, and remineralizing agents in oral care cosmetics: a review of the current situation. Nanomaterials (Basel) 2020;10(1):1-32

12 Turner IG, Ceramics and glasses. In: Narayan R, ed. Biomedical Materials. New York, NY, USA: Springer Science + Business Media; 2009;3-39
13 Walsh T, Worthington HV, Glenny AM, Marinho VC, Jeroncic A. Fluoride toothpastes of different concentrations for preventing dental caries. Cochrane Database Syst Rev 2019;3:CD007868

14 Tomaz PLS, Sousa LA, Aguiar KF, et al. Effects of 1450-ppm fluoride-containing toothpastes associated with boosters on the enamel remineralization and surface roughness after cariogenic challenge. Eur J Dent 2020;14(1):161-170

15 Vilhena FV, Polassi MR, Paloco EAC, Alonso RC, Guiraldo RD, D'Alpino PH. Effectiveness of toothpaste containing REFIX technology against dentin hypersensitivity: a randomized clinical study. J Contemp Dent Pract 2020;21(6):609-614.

16 Buskes JA, Christoffersen J, Arends J. Lesion formation and lesion remineralization in enamel under constant composition conditions. A new technique with applications. Caries Res 1985;19(6):490-496

17 Lagerweij MD, ten Cate JM. Acid susceptibility at various depths of pH-cycled enamel and dentine specimens. Caries Res 2006;40(1):33-37

18 Moron BM, Miyazaki SS, Ito N, et al. Impact of different fluoride concentrations and $\mathrm{pH}$ of dentifrices on tooth erosion/ abrasion in vitro. Aust Dent J 2013;58(1):106-111

19 Raafat Abdelaziz R, Mosallam RS, Yousry MM. Tubular occlusion of simulated hypersensitive dentin by the combined use of ozone and desensitizing agents. Acta Odontol Scand 2011;69(6):395-400

20 Deng M, Wen HL, Dong XL, et al. Effects of 45S5 bioglass on surface properties of dental enamel subjected to $35 \%$ hydrogen peroxide. Int J Oral Sci 2013;5(2):103-110

21 Carvalho SM, Moreira CDF, Oliveira ACX, Oliveira AA, Lemos EMF, Pereira MM, Bioactive glass nanoparticles for periodontal regeneration and applications in dentistry. In: Subramani K, Ahmed W, eds. Nanobiomaterials in Clinical Dentistry. 2nd ed. Elsevier; Oxford, United Kingdom: 2019 351-383

22 Moreno EC, Kresak M, Zahradnik RT. Fluoridated hydroxyapatite solubility and caries formation. Nature 1974;247(5435):64-65

23 Hong Z, Merino EG, Reis RL, Mano JF. Novel rice-shaped bioactive ceramic nanoparticles. Adv Eng Mater 2009;11:B25-B29

24 Parker AS, Patel AN, Al Botros R, et al. Measurement of the efficacy of calcium silicate for the protection and repair of dental enamel. J Dent 2014;42(Suppl 1) :S21-S29

25 Dai Z, Liu M, Ma Y, et al. Effects of fluoride and calcium phosphate materials on remineralization of mild and severe white spot lesions. BioMed Res Int 2019;2019:1271523

26 Gibson IR, Best SM, Bonfield W. Chemical characterization of silicon-substituted hydroxyapatite. J Biomed Mater Res 1999;44(4):422-428

27 Surmeneva MA, Mukhametkaliyev TM, Tyurin AI, et al. Effect of silicate doping on the structure and mechanical properties of thin nanostructured RF magnetron sputter-deposited hydroxyapatite films. Surf Coat Tech 2015;275:176-184

28 Onwubu SC, Mdluli PS, Singh S, Bharuth V, Makgobole MU. Evaluation of the occluding characteristics of nanosized eggshell/titanium dioxide with or without saliva. Eur J Dent 2019;13(4):547-555

29 Peumans M, Politano G, Van Meerbeek B. Treatment of noncarious cervical lesions: when, why, and how. Int J Esthet Dent 2020;15(1):16-42

30 Tian L, Peng C, Shi Y, et al. Effect of mesoporous silica nanoparticles on dentinal tubule occlusion: an in vitro study using SEM and image analysis. Dent Mater J 2014;33(1):125-132

31 West NX, Hughes JA, Addy M. Dentine hypersensitivity: the effects of brushing toothpaste on etched and unetched dentine in vitro. J Oral Rehabil 2002;29(2):167-174

32 Amaechi BT, Lemke KC, Saha S, Gelfond J. Clinical efficacy in relieving dentin hypersensitivity of nanohydroxyapatite-containing cream: A randomized controlled trial. Open Dent J 2018;12:572-585 